\title{
Altered Blood-Brain Barrier and Blood-Spinal Cord Barrier Dynamics in Amyotrophic Lateral Sclerosis: Impact on Medication Efficacy and Safety
}

\author{
Yijun $\operatorname{Pan}^{1}$ and Joseph Nicolazzo ${ }^{1}$ \\ ${ }^{1}$ Monash Institute of Pharmaceutical Sciences, Monash University
}

September 29, 2021

\begin{abstract}
The access of drugs into the central nervous system (CNS) is regulated by the blood-brain barrier (BBB) and blood-spinal cord barrier (BSCB). A large body of evidence supports perturbation of these barriers in neurodegenerative diseases, including Alzheimer's disease and Parkinson's disease. Modifications to the BBB and BSCB are also reported in amyotrophic lateral sclerosis (ALS), albeit these modifications have received less attention relative to those in other neurodegenerative diseases. Such alterations to the BBB and BSCB have the potential to impact on CNS exposure of drugs in ALS, modulating the effectiveness of drugs intended to reach the brain and the toxicity of drugs that are not intended to reach the brain. Given the clinical importance of these phenomena, this review will summarise reported modifications to the BBB and BSCB in ALS, discuss their impact on CNS drug exposure and suggest further research directions so as to optimise medicine use in people with ALS.
\end{abstract}

\section{Altered Blood-Brain Barrier and Blood-Spinal Cord Barrier Dynamics in Amyotrophic Lateral Sclerosis: Impact on Medication Efficacy and Safety}

Yijun Pan, Joseph A Nicolazzo*

Drug Delivery, Disposition and Dynamics, Monash Institute of Pharmaceutical Sciences, Monash University, 399 Royal Parade, Parkville, Victoria 3052, Australia.

* Corresponding author

Ph: +61 39903 9605, Fax: +61399039583

E-mail: joseph.nicolazzo@monash.edu

\section{LIST OF ABBREVIATIONS}

\begin{tabular}{ll}
\hline ABC proteins & ATP-binding cassette (ABC) proteins \\
\hline AD & Alzheimer's disease \\
ALS & amyotrophic lateral sclerosis \\
BBB & blood-brain barrier \\
BCRP & breast cancer resistance protein \\
BSCB & blood-spinal cord barrier \\
CNS & central nervous system \\
GLUT1 & glucose transporter 1 \\
JAM & junctional adhesion molecule \\
LAT1 & L-type amino acid transporter 1
\end{tabular}




\begin{tabular}{ll}
\hline ABC proteins & ATP-binding cassette (ABC) proteins \\
\hline PD & Parkinson's disease \\
P-gp & P-glycoprotein \\
SOD1 & superoxide dismutase 1 \\
TDP43 & TAR DNA-binding protein 43 \\
TJ & tight junction \\
ZO & zonula occludens \\
\hline
\end{tabular}

\begin{abstract}
The access of drugs into the central nervous system (CNS) is regulated by the blood-brain barrier (BBB) and blood-spinal cord barrier (BSCB). A large body of evidence supports perturbation of these barriers in neurodegenerative diseases, including Alzheimer's disease and Parkinson's disease. Modifications to the $\mathrm{BBB}$ and BSCB are also reported in amyotrophic lateral sclerosis (ALS), albeit these modifications have received less attention relative to those in other neurodegenerative diseases. Such alterations to the BBB and BSCB have the potential to impact on CNS exposure of drugs in ALS, modulating the effectiveness of drugs intended to reach the brain and the toxicity of drugs that are not intended to reach the brain. Given the clinical importance of these phenomena, this review will summarise reported modifications to the BBB and BSCB in ALS, discuss their impact on CNS drug exposure and suggest further research directions so as to optimise medicine use in people with ALS.
\end{abstract}

\title{
Introduction
}

Amyotrophic lateral sclerosis (ALS) is the most common motor neurone disease in adults, leading to muscle weakness and eventual paralysis and respiratory failure. ALS typically occurs in late middle life (51-66 years), with an incidence of 0.6-3.8 per 100000 person-years and a prevalence of 4.1-8.4 per 100000 persons (Longinetti \& Fang, 2019). Despite affecting individuals for more than 150 years, this progressive neurodegenerative and fatal disease has limited treatment options, with riluzole and edaravone being the only two United States Food and Drug Administration-approved treatment options (Shefner et al., 2020). The majority of individuals with ALS can survive 3-5 years after appearance of symptoms, despite being administered riluzole (Chiò et al., 2009). ALS is mainly a sporadic disease with about $10 \%$ of cases being familial in nature (Chiò et al., 2009). Multiple factors contribute to the pathogenesis of ALS, including dysfunctional RNA metabolism, defective protein homeostasis, mitochondrial dysfunction, oxidative stress, neuroinflammation, and vesicular transport defects (Mejzini, Flynn, Pitout, Fletcher, Wilton \& Akkari, 2019).

Despite a wealth of research being undertaken to reverse the pathology associated with ALS, translating this to ALS therapeutics has not been very successful. While there are multiple reasons for preclinical-to-clinical failure for ALS therapeutics, including heterogeneity of ALS pathology (Beghi et al., 2007) and species differences in efficacy, a major reason as to why many CNS candidates fail to reach the market is their inability to reach their site of action within the brain, due to the defence nature of the CNS barriers (Nicolazzo, Charman \& Charman, 2006). These barriers, including the blood-brain barrier (BBB) and blood-spinal cord barrier (BSCB), consist of a layer of endothelial cells connected by tight junction (TJ) proteins to limit paracellular transport and express influx and efflux transporters that precisely control permeation of circulating solutes including drugs (Abbott, Patabendige, Dolman, Yusof \& Begley, 2010). In neurodegenerative disorders such as Alzheimer's disease (AD) and Parkinson's disease (PD), there are multiple reports of altered BBB ultrastructure and permeability, which have been reported to affect CNS drug delivery (Pan \& Nicolazzo, 2018).

Modifications to the BBB and BSCB are also reported in ALS and such modifications have the potential to impact on CNS exposure of drugs, including those that are intended to reach the brain and those for which CNS exposure leads to off-target effects. As well as riluzole and/or edaravone, individuals with ALS are prescribed 5-10 medicines intended to reach the brain for management of common ALS symptoms such as 
cramps, spasticity and pain (Meyer et al., 2020). In the last 12 months of life, $57.4 \%$ and $44.1 \%$ of individuals with ALS are prescribed psychoanaleptics and psycholeptics, respectively (Grande, Morin, Vetrano, Fastbom \& Johnell, 2017), and any BBB/BSCB changes could impact on their CNS exposure and efficacy. In addition, individuals with ALS are often afflicted with various comorbidities including hypertension, diabetes and hypercholesterolaemia requiring medicines that do not require access into the brain (Hobson \& McDermott, 2016). Any alterations to the BBB/BSCB in ALS could result in undesirable CNS accumulation of these drugs, leading to unintended neurotoxicity in people with ALS.

Therefore, characterising the status of the CNS barriers in ALS is important for both drug discovery and for optimising medicine use in individuals with ALS. A better characterisation of the status of the BBB/BSCB can guide the design of CNS barrier-targeting approaches to enhance CNS access of novel preclinical candidates for ALS. On the other hand, appreciating the status of the CNS barriers in ALS could assist in implementing strategies to minimise the CNS exposure of drugs not intending to reach the brain, so as to minimise undesirable adverse effects and ultimately improving quality of life. This review will provide a general introduction to the structure and function of CNS barriers, highlight modifications of CNS barriers in ALS and discuss how these modifications have the potential to impact on drug development and medication safety and effectiveness in ALS.

\section{The CNS barriers}

\section{$2.1 \mathrm{BBB}$ and BSCB physiology}

The CNS barriers are formed by a layer of endothelial cells separating the blood and the CNS parenchyma (Figure 1 ), with the BBB and BSCB protecting the brain and spinal cord, respectively, from bloodderived toxins. The endothelial cells are connected by inter-endothelial TJ and adhesion proteins. A basement membrane supports the brain or spinal cord endothelial cell and associated pericytes, with astrocytic end-feet ensheathing the basement membrane (Abbott, Patabendige, Dolman, Yusof \& Begley, 2010) (Figure 1 ).

Figure 1. A schematic diagram illustrating the constituents of the CNS barriers. The endothelial cells are connected by ti

The defence characteristics of the CNS barriers can be considered as both physical and biochemical barriers. The physical barrier is imparted by TJs, which are formed through a sophisticated network of interacting proteins, such as occludin, claudins, junctional adhesion molecules (JAMs) and zonula occludens, which prevent the paracellular movement of solutes (Abbott, Patabendige, Dolman, Yusof \& Begley, 2010). The biochemical barrier is imparted by the function of efflux transporters, such as the ATP-binding cassette $(\mathrm{ABC})$ proteins, P-glycoprotein ( $\mathrm{P}-\mathrm{gp})$ and breast cancer resistance protein $(\mathrm{BCRP})$, that effectively exclude various endogenous and exogenous toxins from the endothelial cells. In addition to the physical and biochemical barriers, there exist a number of influx transporters, such as glucose transporter 1 (GLUT1) and large amino acid transporter (LAT1), which facilitate the uptake of essential nutrients into the CNS (Abbott, Patabendige, Dolman, Yusof \& Begley, 2010). The BSCB is slightly more permeable than the BBB in mice (Pan, Banks \& Kastin, 1997), likely attributed to reduced abundance of zonula occludens-1 and occludin (Ge \& Pachter, 2006). Furthermore, the levels of efflux transporters and TJ proteins were reported higher in the human BBB relative to the BSCB (Uchida et al., 2020).

\subsection{CNS barriers and drug delivery}

The presence of efflux drug transporters such as P-gp and BCRP at CNS barriers, in addition to the TJ architecture, are known to limit the CNS access of drugs, reducing their ability to reach their site of action within the brain (Nicolazzo, Charman \& Charman, 2006). Pharmacological inhibition of P-gp has been trialled as an approach to improve CNS drug delivery; however, this approach has not been clinically-translated due to the undesirable effects associated with blanket suppression of P-gp function (Davis, Sanchez-Covarubias \& Tome, 2014). On the other hand, influx transporters have been targeted to improve CNS drug delivery. For example, drugs and prodrugs (e.g. L-DOPA, melphalan, and gabapentin) 
have been designed to be recognised as substrates to LAT1 (Puris, Gynther, Auriola \& Huttunen, 2020), leading to improved brain uptake and effectiveness.

While CNS barriers are considered a major barrier to drug delivery under healthy conditions, it is becoming increasingly apparent that these barriers are modulated in various diseases of the CNS, such as AD and PD (Pan \& Nicolazzo, 2018). This has been shown to lead to altered drug access to the CNS, potentially resulting in a loss of desirable pharmacological effects or an increased risk of CNS adverse effects. While there has been significant focus on CNS barrier modifications in AD and PD (Al-Bachari, Naish, Parker, Emsley \& Parkes, 2020; Sweeney, Sagare \& Zlokovic, 2018), less studies have focused on modification to the CNS barriers in ALS. Therefore, this review will (i) provide a contemporary update on the status of the BBB and BSCB in ALS, (ii) provide insight into the potential impact of these changes on CNS drug exposure and medicine use in people with ALS, and (iii) suggest recommendations for future research directions to ensure effective CNS drug delivery and medicine use in ALS.

\section{Modification of CNS barriers in ALS}

Many of the studies reporting altered CNS barrier function in sporadic ALS are based on observations using post mortem human tissues, while transgenic rodent models expressing mutant genes, e.g. superoxide dismutase 1 G93A (SOD1 ${ }^{\mathrm{G} 93 \mathrm{~A}}$ ) have significantly contributed to our understanding of CNS barrier changes in familial ALS (summarised in Table 1, Table 2 and graphically presented in Figure 2 ).

\subsection{Evidence from post-mortem human tissues}

\subsubsection{Ultrastructural modifications}

In postmortem ALS human spinal cord, electron microscopy and immunohistochemistry revealed swelling and cytoplasmic vacuolisation of microvascular endothelial cells, degeneration of pericytes and detachment of astrocyte end-feet processes from endothelial cells (Garbuzova-Davis et al., 2012; Miyazaki et al., 2011; Sasaki, 2015; Yamadera et al., 2015). Studies also reported increased (Garbuzova-Davis et al., 2012; Waters et al., 2021) or decreased (Miyazaki et al., 2011; Ono et al., 1998) collagen content in the basement membrane of the spinal cord microvasculature in ALS patients. Reduced collagen content in the basement membrane can be to due cellular damage, whereas a thickened basement membrane could be a result of repetitive regeneration. This discrepancy in the above reports could be attributed to the different CNS regions assessed, the heterogeneity in ALS pathology, and varied quantification methods employed to assess collagen content. Further studies are required to confirm this in a more systematic manner, and assess the impact of basement thickening or thinning on CNS exposure of passively-diffusing compounds, given that brain microvascular basement membrane thickening has been associated with reduced BBB transport of passively-diffusing drugs in a mouse model of AD (Mehta, Short \& Nicolazzo, 2013).

\subsubsection{Reduced expression of TJ and adhesion proteins}

Decreased expression of TJ and adhesion proteins in spinal cords from ALS patients has been confirmed using PCR and Western Blot. Henkelet al. assessed mRNA expression of zonula occludens-1, occludin, and claudin-5 in lumbar spinal cord homogenates from individuals with ALS (30 sporadic and 4 familial cases) and 16 non-ALS controls (Henkel, Beers, Wen, Bowser \& Appel, 2009). A significant reduction in zonula occludens-1 and occludin levels were identified in sporadic ALS and familial ALS, respectively, while claudin-5 was unchanged in both sporadic and familial ALS. In another study, a significant decrease in most TJ and adhesions proteins were reported in ALS spinal cord (cervical and lumbar) relative to non-ALS controls, including zonula occludens-1, occludin, claudin-5, JAM-1 and VE-cadherin (Garbuzova-Davis et al., 2012). Despite decreased TJ protein expression at the ALS BSCB, the junction morphology was generally well-preserved as assessed by electron microscopy (Sasaki, 2015). Based on these TJ and adhesion junction reductions, it would be predicted that spinal cord access of molecules would be increased as a result of increased paracellular permeability.

\subsubsection{Leaky CNS barriers}


For human post mortem studies, the leakiness of CNS barriers is often inferred from the presence of plasmaderived proteins in brain or spinal cord. Donnenfeld et al. identified IgG and $\mathrm{C} 3 / \mathrm{C} 4$ complement in the motor cortex and spinal cord of ALS patients nearly 40 years ago (Donnenfeld, Kascsak \& Bartfeld, 1984). These deposits were detected immunohistochemically in 5 of 13 ALS motor cortices and 6 of 16 ALS spinal cords, but not in non-ALS controls. Findings by Donnenfeldet al. were later confirmed by Engelhardt et al . immunohistochemically, where IgG was identified in 13 of 15 ALS spinal cords and 6 of 11 ALS motor cortices (Engelhardt \& Appel, 1990). In addition to IgG, Winker et al. noted haemoglobin, fibrinogen and thrombin in the ALS cervical spinal cord (8 sporadic ALS cases, 3 familial ALS cases) but not in nonALS controls (5 cases) (Winkler, Sengillo, Sullivan, Henkel, Appel \& Zlokovic, 2013). In line with these postmortem findings, increased IgG (1.26-fold) and albumin (1.28-fold) has been reported by Leonardi et al. (Leonardi, Abbruzzese, Arata, Cocito \& Vische, 1984) in ALS cerebrospinal fluid (CSF) (90 ALS cases, 50 controls) and elevated haemoglobin levels (1.86-fold) has been reported in the CSF of individuals with ALS by Waters et al. (236 ALS cases, 87 controls) (Waters et al., 2021). Consistently, these studies demonstrate that CNS barrier permeability is generally increased in individuals with ALS, which is very likely a result of the ultrastructural abnormality and reduced expression of TJs at the ALS CNS barriers. While these modifications likely contribute to the leakiness of endogenous proteins into the CNS, the impact of these changes on the CNS access of small and large molecular weight therapeutics has yet to be undertaken clinically. This could be achieved using imaging techniques such as magnetic resonance imaging to visualise CNS access of gadolinium in ALS.

\subsubsection{Increased expression of P-gp and BCRP}

The cellular distribution and expression of P-gp and BCRP has been assessed in the cervical spinal cord and motor cortex obtained from individuals with ALS. Using immunohistochemistry, van Vliet et al. has demonstrated a dramatic ( $\sim 30$-fold) increase in P-gp abundance in cervical spinal cord and motor cortex astrocytes in ALS tissues relative to control specimens, while P-gp abundance at the endothelial cell lining of microvessels was comparable between control and ALS spinal cord and motor cortex specimens (van Vliet et al., 2019). A similar study was conducted by Jablonski et al. , who reported an elevated P-gp abundance in microvessels from ALS lumbar spinal cord (Jablonski et al., 2012). The discrepancies between these studies could be due to different CNS regions assessed (cervical spinal cord, lumbar spinal cord, and motor cortex). In terms of BCRP, microvasculature expression was observed in both control and ALS specimens, and its abundance did not differ between control and ALS in the cervical spinal cord but was marginally increased in the ALS motor cortex (van Vliet et al., 2019).

Further studies are required to confirm these findings and possibly improve our understanding of CNS barriers protein expression in ALS, i.e. beyond P-gp and BCRP. Specialised microvascular isolation techniques can be implemented to obtain high purity of microvessels or isolated endothelial cells, which can be analysed via proteomic approaches. Such approaches may identify modified expression of efflux and influx transporters in ALS, which can assist in (i) targeting transporters to increase CNS exposure of otherwise impermeable compounds and (ii) guiding dosage regimen design of medicines which are substrates for such transporters to avoid potential CNS toxicity. Furthermore, the functional consequence of these expression changes on CNS drug exposure in humans is completely lacking, and could be confirmed using positron emission tomography studies with ${ }^{11} \mathrm{C}$-verapamil (as a substrate of $\mathrm{P}$-gp) as has been undertaken in humans with $\mathrm{AD}$ and $\mathrm{PD}$ (Bartels et al., 2008; Lubberink, van Assema, Hendrikse, Schuit, Lammertsma \& Van Berckel, 2010).

Table 1. Evidence of CNS barrier modifications in ALS from human biospecimens.

\begin{tabular}{lll}
\hline Parameter & Observation & References \\
Ultrastructure & morphological changes in & Miyazaki 2011, \\
endothelium, astrocytes, & Garbuzova-Davis 2012, Winkler \\
pericytes & 2013 , Sasaki 2015, Yamadera \\
& | collagen content & Garbuzova-Davis 2012, Sasaki \\
& & 2015, Waters 2021
\end{tabular}


Tight junctions

Leakiness

Efflux transporters P-gp

BCRP
| collagen content

well-preserved structure (electron

microscopy)

| mRNA or protein expression

blood derived protein detected in the spinal cord/cortex/CSF

| (spinal cord homogenates) no change (spinal cord and motor cortex endothelium) | (spinal cord and motor cortex astrocytes)

| in spinal cord homogenates | (motor cortex endothelium); | (spinal cord and motor cortex astrocytes)
Ono 1998, Miyazaki 2011

Sasaki 2015

Henkel 2009, Garbuzova-Davis 2012

Donnefeld 1984, Leonardi 1984, Engelhardt 1990, Garbuzova-Davis 2012, Winkler

2013, Waters 2012

Jablonski 2012

van Vliet 2019

Jablonski 2012

van Vliet 2019

\subsection{Evidence from rodent models}

\subsubsection{Transgenic ALS rodent models used for evaluation of CNS barriers}

In order to provide a review of the changes to CNS barriers in animal models of ALS, it is important to describe the commonly-used models of ALS. The human SOD1 ${ }^{\mathrm{G} 93 \mathrm{~A}}$ transgenic mouse model, which harbors a mutation in human SOD1, was the first established animal model for ALS (Gurney et al., 1994; Rosen et al., 1993), which recapitulates many human ALS symptoms; mice first demonstrate a hindlimb tremor, followed by loss of the hindlimb splaying reflex, and eventually paralysis and death. Numerous SOD1 transgenic models of ALS were designed thereafter using patient sequence variants from genomic fragments, including the G37R mutation (Wong et al., 1995), the G86R mutation (Bartels et al., 2008), the G85R mutation (Bruijn et al., 1997), the L126Z mutation (Wang et al., 2005), and the T116X mutation (Han-Xiang et al., 2008). While each line differs in onset, presentation, and progression, SOD1 overexpressing transgenic mice generally share traits of significant motor neuron loss, axonal denervation, progressive paralysis, and reduced lifespan (Philips \& Rothstein, 2015; Picher-Martel, Valdmanis, Gould, Julien \& Dupré, 2016). In addition to the mouse model, a transgenic SOD1 ${ }^{\mathrm{G} 93 \mathrm{~A}}$ rat model of ALS has been developed and validated, exhibiting a more rapid progression of disease (Howland et al., 2002). However, SOD1 models are based off a small fraction of an extremely heterogeneous disease, as only $\sim 2 \%$ of human ALS (familial and sporadic) is due to SOD1 mutations (Picher-Martel, Valdmanis, Gould, Julien \& Dupré, 2016). Therefore, findings obtained with this model need to be carefully considered as they may not be replicated in the majority of individuals with ALS.

While TAR DNA-binding protein 43 (TDP43) mutations only account for less than $5 \%$ of familial ALS cases, its identification as a causative gene to ALS in 2008 resulted in 20 mouse models (Picher-Martel, Valdmanis, Gould, Julien \& Dupré, 2016). The TDP43 ${ }^{\text {A315T }}$ mouse was the first reported TDP43 mutation-based ALS mouse model (Wegorzewska, Bell, Cairns, Miller \& Baloh, 2009), which develops a gait abnormality, significant weight loss and a characteristic "swimming" gait.

While there exist other mouse models of ALS, such as FUS ${ }^{1-359}$ mice (Shelkovnikova et al., 2013) and C9orf72-based mouse models (Jiang et al., 2016; O'Rourke et al., 2015; Peters et al., 2015), they will not be described in detail as no studies assessing BBB or BSCB function have been reported in these animal models.

\subsubsection{Ultrastructural abnormality}


Similar to individuals with ALS, altered ultrastructure of CNS barriers has been reported in SOD1 G93A mice (Garbuzova-Davis, Haller, Saporta, Kolomey, Nicosia \& Sanberg, 2007). Brain stem and cervical and lumbar spinal cords of SOD1 ${ }^{\mathrm{G} 93 \mathrm{~A}}$ mice were assessed using electron microscopy, and degenerating endothelial cells, vacuolated endothelial cytoplasm, and swollen astrocyte end-feet were noted at an early disease stage that worsened with disease progression. A thickened basement membrane was noted around the severely degenerated endothelia of all three regions assessed, suggesting a reparative process (Garbuzova-Davis, Haller, Saporta, Kolomey, Nicosia \& Sanberg, 2007). In their subsequent study, reduced laminin-1 (major noncollagenous basement membrane glycoprotein) was demonstrated immunohistochemically in the BSCB of SOD1 ${ }^{\text {G93A }}$ mice at both early and late stages (Garbuzova-Davis et al., 2007). In line with this, reduced and undetectable collagen IV content was reported in the BSCB basement membrane of SOD1 ${ }^{\mathrm{G} 93 \mathrm{~A}}$ mice (Miyazaki et al., 2011). These ALS-related modifications at the BSCB were also identified in SOD1G93A rats (Nicaise et al., 2009). Despite progression of ALS pathology being more rapid in the SOD1 ${ }^{\mathrm{G} 93 \mathrm{~A}}$ rat model, ultrastructural alterations were only observed in symptomatic SOD1 ${ }^{\mathrm{G} 93 \mathrm{~A}}$ rats but not pre-symptomatic rats. Overall, these studies suggest a compromised BSCB structural integrity in SOD1 ${ }^{\text {G93A }}$ models, and that this may lead to increased penetration of drugs into the spinal cord, however, functional studies to confirm this hypothesis are lacking. Furthermore, little information is available on any potential ultrastructural changes at the $\mathrm{BBB}$ of the motor cortex in $\mathrm{SOD} 1^{\mathrm{G} 93 \mathrm{~A}}$ mice, an area which is important to investigate to appreciate the impact of the disease on brain access of drugs.

\subsubsection{Reduced expression of TJ proteins}

Using electron microscopic analysis, Garbuzova-Davis et al.suggested that the endothelial cell TJs appeared intact in early and late symptomatic SOD1 ${ }^{\text {G93A }}$ mice (Garbuzova-Davis, Haller, Saporta, Kolomey, Nicosia \& Sanberg, 2007). However, using a more quantitative approach (Western blot), a 30-60\% reduction in zonula occludens-1, occludin and claudin-5 was consistently observed in spinal cord microvessels of SOD1 ${ }^{\text {G93A }}$ mice relative to wildtype mice (Zhong et al., 2008). In addition, a progressive reduction in occludin abundance at the BSCB in SOD1 ${ }^{\mathrm{G} 93 \mathrm{~A}}$ mice was demonstrated by Miyazaki et al. using immunohistochemistry and Western blot (Miyazaki et al., 2011). Similarly, in SOD1 ${ }^{\text {G93A }}$ rats, zonula occludens-1 and occludin mRNA were significantly reduced in the lumbar spinal cords at the symptomatic stage only but not in the brain or brain stem (Nicaise et al., 2009).

\subsubsection{Leaky CNS barriers}

An increased paracellular permeability of the BSCB was clearly demonstrated by intravenous injection of Evans Blue, a tracer used to assess barrier disruption, in SOD1 G93A mice (Garbuzova-Davis et al., 2007). Vascular leakage (indicated by Evans Blue extravasation) was observed in lumbar spinal cord, and cervical spinal cord from 13 weeks of age in SOD1 ${ }^{\mathrm{G} 93 \mathrm{~A}}$ mice. Considerable vessel permeability was observed in early as well as late stage symptomatic SOD $1^{\mathrm{G} 93 \mathrm{~A}}$ mice. Notably, more vascular leakage was detected in the lumbar spinal cord than cervical spinal cord at the late stage of disease. Using Evans Blue again, vessel leakiness has been demonstrated in the spinal cord and brain stem, but not the brain, of SOD1 ${ }^{\text {G93A }}$ rats at symptomatic stages (Nicaise et al., 2009). However, using a sensitive magnetic resonance imaging approach, increased BBB permeability was demonstrated in the brains of SOD1 ${ }^{\text {G93A }}$ rats (Andjus et al., 2009). Overall, these studies demonstrate increased permeability of CNS barriers in rodent models of ALS, which is expected based on reduced TJ expression in these models. However, whether such changes lead to altered drug penetration into the spinal cord or brain of ALS mice remains to be investigated. It would be hypothesised that drug penetration would increase in mouse models of ALS based on the reported TJ dysfunction and paracellular, however, the modifications in efflux transporter expression (described below) may counteract this leakiness, and indeed reduce CNS exposure of drugs.

\subsubsection{Increased expression and activity of P-gp and BCRP}

A selective increase in microvascular expression of P-gp and BCRP during disease progression in three ALS mouse models (SOD1 ${ }^{\mathrm{G} 93 \mathrm{~A}}$, SOD1 ${ }^{\mathrm{G} 86 \mathrm{R}}$ and TDP43 ${ }^{\mathrm{A} 315 \mathrm{~T}}$ ) has been reported in the cerebral cortex and spinal cord (Jablonski et al., 2012; Milane et al., 2010). mRNA levels were also significantly increased in lumbar 
spinal cord homogenates 2.13-fold for P-gp and 1.72-fold for BCRP in mice at symptomatic stages compared to those at presymptomatic stages (Jablonski et al., 2012). In addition, Western blot was employed to assess P-gp and BCRP abundance in the whole spinal cord and cerebral cortex of SOD1G93A mice. Increased expression of P-gp (1.96-fold) and BCRP (1.69-fold) was observed in the spinal cord of symptomatic mice compared to presymptomatic SOD1 ${ }^{\text {G93A }}$ mice and elevated levels of P-gp (1.35-fold) and BCRP (1.28-fold) were reported in the cerebral cortex of symptomatic mice compared to presymptomatic SOD1 ${ }^{\mathrm{G} 93 \mathrm{~A}}$ mice (Jablonski et al., 2012). The increased expression of P-gp and BCRP was demonstrated in another mouse model of ALS by Jablonski et al. comparing P-gp and BCRP levels in the spinal cord and cerebral cortex of symptomatic TDP43 ${ }^{\mathrm{A} 315 \mathrm{~T}}$ mice with presymptomatic mice. An independent group (Milane et al., 2010) demonstrated increased P-gp abundance (1.5-fold) in microvessels isolated from the brains of presymptomatic $\mathrm{SOD} 1^{\mathrm{G} 86 \mathrm{R}}$ mice compared to age-matched wildtype controls. However, no alteration of BCRP abundance was detected in these SOD1 ${ }^{\mathrm{G} 86 \mathrm{R}}$ mice.

In SOD1 ${ }^{\text {G93A }}$ rats, microvessels from brain and spinal cord sections were assessed immunohistochemically and microvessels were also isolated for protein quantification using Western blot (Chan, Evans, Banks, Mesev, Miller \& Cannon, 2017). In this study Chanet al . demonstrated immunohistochemically that P-gp protein expression was significantly increased in the cerebral cortex (1.88-fold) and the spinal cord (1.46-fold) from symptomatic SOD1 ${ }^{\mathrm{G} 93 \mathrm{~A}}$ rats compared to age-matched wildtype rats. In addition, the expression of BCRP was not affected when assessed immunohistochemically. Given immunohistochemistry is semi-quantitative, protein expression in isolated microvessels was reassessed using Western blot, and a 1.5-fold increase in P-gp expression was demonstrated in isolated microvessels from brain and spinal cord of symptomatic SOD1 ${ }^{\mathrm{G} 93 \mathrm{~A}}$ rats. It was also noted that microvascular BCRP expression increased 1.15- and 1.25-fold in brain and spinal cord, respectively, although statistical significance was not achieved. These studies clearly demonstrate increased expression of P-gp and sometimes BCRP in the brain and spinal cord in models of ALS. Some differences in the results were observed. For example, whole brain and spinal cord homogenates were used by Jablonski et al . and the samples obtained from the microvascular isolation techniques employed by Chanet al. and Milane et al. often contain astrocytes, which express P-gp (Golden \& Pardridge, 1999).

In addition to assessing expression of P-gp and BCRP, Jablonski et al. assessed their activity using a confocal microscopy-based transport assay where microvessles were incubated with fluorescent substrates (NBDcyclosporin A for P-gp; bodipy-prazosin for BCRP) (Jablonski et al., 2012). Luminal substrate accumulation was measured in the absence and presence of specific transport inhibitors (PSC833 for P-gp, Ko143 for BCRP). This assay successfully demonstrated that the transport activity of P-gp was increased 1.8-fold and 2 -fold in brain and spinal cord microvessels, respectively, from symptomatic SOD1 ${ }^{\mathrm{G} 93 \mathrm{~A}}$ mice relative to agematched wildtype mice and presymptomatic SOD1 ${ }^{\mathrm{G} 93 \mathrm{~A}}$ mice. In a follow up study, the in vivo accumulation of a specific P-gp substrate, LD800, in the spinal cord of symptomatic SOD1 ${ }^{\text {G93A }}$ mice was assessed and compared to wildtype mice following intraperitoneal administration (Jablonski et al., 2014). Spinal cord levels of LD800 levels were significantly lower in SOD1 ${ }^{\mathrm{G} 93 \mathrm{~A}}$ mice comparing to wildtype mice, suggesting increased P-gp activity at the BSCB, in line with increased P-gp expression. A similar study was performed, where a P-gp substrate $\left[{ }^{3} \mathrm{H}\right]$-digoxin and a BCRP substrate $\left[{ }^{3} \mathrm{H}\right]$-prazosin were dosed intraperitoneally to both SOD1 ${ }^{\mathrm{G} 86 \mathrm{R}}$ mice and wildtype mice (Milane et al., 2010). A reduced disposition of $\left[{ }^{3} \mathrm{H}\right]$-digoxin (1.5-fold) but not $\left[{ }^{3} \mathrm{H}\right]$-prazosin was observed in the brain of presymptomatic SOD1 ${ }^{\mathrm{G} 86 \mathrm{R}}$ mice compared to wildtype controls, suggesting increased function of P-gp but not BCRP in this mouse model. This alteration is in line with reduced P-gp protein abundance (1.5-fold) and unchanged BCRP abundance observed in this mouse model.

Chan et al. demonstrated a 2-fold increase in P-gp activity in microvessels isolated from the brain and spinal cord of symptomatic SOD1 ${ }^{\mathrm{G} 93 \mathrm{~A}}$ rats compared to those from presymptomatic rats using the same confocal microscopy-based transport assay mentioned above (Chan, Evans, Banks, Mesev, Miller \& Cannon, 2017). Overall, these studies demonstrate increased P-gp and BCRP abundance and activities at the CNS barriers in ALS rodent models, suggesting that the barriers may be more restrictive to substrates of these transporters. This is of particular concern given that riluzole, which is effective in early ALS and less effective in late stage ALS, is a substrate of P-gp and BCRP (Zoccolella et al., 2007). The reduced effectiveness of riluzole 
in SOD1 ${ }^{\mathrm{G} 93 \mathrm{~A}}$ rats may result from $\mathrm{P}-\mathrm{gp} / \mathrm{BCRP}$ overactivity, as less riluzole may be able to enter the CNS. In fact, a 1.7-fold reduction in riluzole disposition into the brain of SOD1 ${ }^{\mathrm{G} 86 \mathrm{R}}$ has been reported compared to wildtype mice following intraperitoneal administration (Milane et al., 2010). Therefore, approaches to overcome increased P-gp and BCRP activities in ALS may improve the effectiveness of riluzole. This has been confirmed in SOD1 ${ }^{\text {G93A }}$ mice that received riluzole with or without elacridar (an inhibitor of P-gp and BCRP) (Jablonski et al., 2014). The efficacy of riluzole improved when co-administered with elacridar and this was associated with increased riluzole delivery to the brain. However, such generic inhibition of P-gp/BCRP comes with side effects, and therefore, an approach to restore P-gp/BCRP to levels similar to the healthy CNS barriers is more likely to be a more effective clinical strategy. In addition, while much has been reported on P-gp and BCRP in ALS, our knowledge on other transporters at the CNS barriers is limited. Profiling the expression at the CNS barriers could potentially identify efflux transporters that should be avoided for effective CNS delivery or influx transporters, which could be effectively targeted to increase CNS exposure of drugs intended to reach the CNS.

Table 2. Evidence of CNS barrier modifications in ALS from rodent models.

\begin{tabular}{|c|c|c|}
\hline Parameter & Observation & References \\
\hline \multirow[t]{2}{*}{ Ultrastructure } & $\begin{array}{l}\text { morphological changes to } \\
\text { endothelium, astrocytes, and } \\
\text { pericytes }\end{array}$ & $\begin{array}{l}\text { Garbuzova-Davis 2007a, Nicaise } \\
2009\end{array}$ \\
\hline & $\begin{array}{l}\text { structural proteins in the } \\
\text { basement membrane (e.g. } \\
\text { collagen IV, laminin-1) }\end{array}$ & $\begin{array}{l}\text { Garbuzova-Davis 2007b, } \\
\text { Miyazaki 2011, Nicaise } 2009\end{array}$ \\
\hline Tight junctions & mRNA or protein expression & $\begin{array}{l}\text { Miyazaki 2011, Zhong 2008, } \\
\text { Nicaise } 2009\end{array}$ \\
\hline Leakiness & | Evans Blue extravasation & $\begin{array}{l}\text { Garbuzova-Davis 2007b, Nicaise } \\
2009\end{array}$ \\
\hline Efflux transporter P-gp & | expression and activity & $\begin{array}{l}\text { Milane 2010, Jablonski 2012, } \\
\text { Jablonski 2014, Chan } 2017\end{array}$ \\
\hline \multirow[t]{2}{*}{ BCRP } & $\begin{array}{l}\text { no change to expression and } \\
\text { activity }\end{array}$ & Milane 2010, Chan 2017 \\
\hline & expression and activity & Jablonski 2012 \\
\hline
\end{tabular}

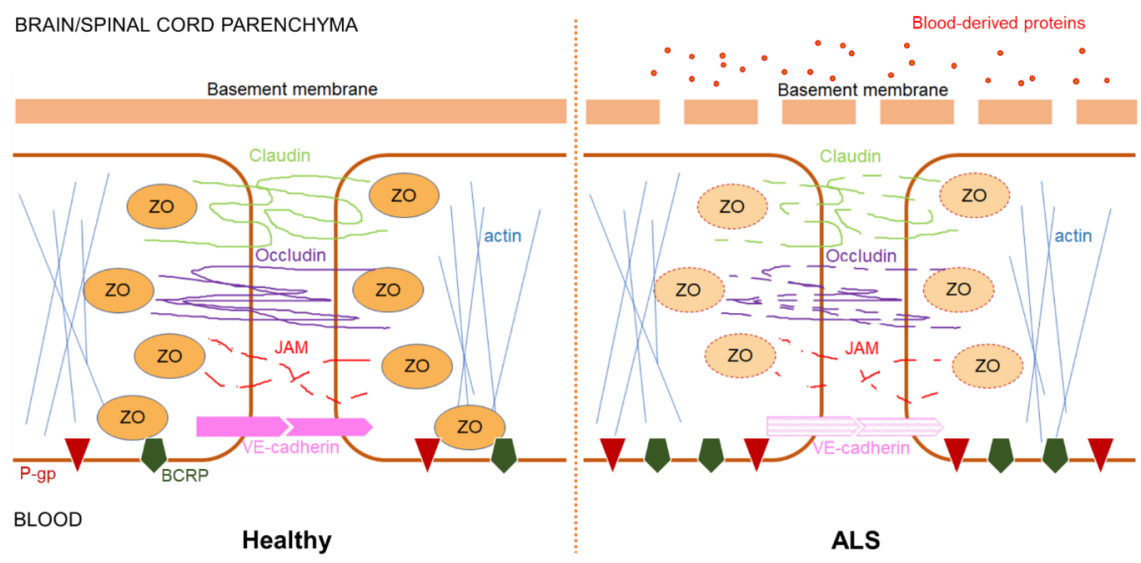

Figure 2 . A summary of the modifications to TJ, adhesion junctions and efflux transporter expression at ALS CNS barriers. The abundance of TJ and adhesion proteins are generally reduced, leading to a compromised junctional integrity and increase in paracellular permeability. The basement membrane thickness 
is reported to either increase as a reparative mechanism or decrease with reduced collagen and laminin in ALS, with discrepancies reported in human tissues, while being consistently decreased in animal models. The expression of P-gp and BCRP are generally increased in ALS, which is expected to result in reduced CNS exposure of drugs that are substrates for these efflux transporters. ZO: zonula occludens, P-gp: P-glycoprotein, BCRP: breast cancer resistance protein, JAM: junctional adhesion molecules.

\section{Summary and future directions}

Human and rodent studies have consistently demonstrated ultrastructural abnormality, increased paracellular permeability and elevated P-gp (and sometimes BCRP) expression and activity at the CNS barriers in ALS. Based on the reports of reduced TJ function, it would be expected that general CNS permeability to drugs would be elevated in ALS, however, this may be counteracted especially if the drugs are substrates of Pgp and/or BCRP. Furthermore, if there is indeed a thickening of the cerebral or spinal microvasculature basement membrane, this could result in lower brain and spinal cord access of drugs as has been reported in a mouse model of AD with thickened cerebrovascular basement membrane (Mehta, Short \& Nicolazzo, 2013). Therefore, it is clear that a more detailed functional analysis of transport processes of many drugs which are trafficked via different mechanisms (i.e. paracellular, transcellular, substrates of influx and efflux transporters) is required so as to predict how CNS access of drugs indeed alters in ALS.

With disease progression, the expression and activity of P-gp and BCRP generally increases, which may lead to suboptimal drug delivery, while this yet to be confirmed in people with ALS, for example, by using PET imaging. If this is validated in humans, specialised approaches can be trialled to improve the CNS access of riluzole (and other CNS-acting drugs) to improve therapeutic outcomes. This can be via pharmacological manipulation of P-gp expression and activity or by transiently disrupting the CNS barriers using emerging technology such as MR-guided focused ultrasound (Abrahao et al., 2019).

Our current understanding of the CNS barriers in ALS is still limited. The majority of studies have only investigated P-gp and BCRP expression. Proteomic studies can be performed using microvessels isolated from post-mortem ALS human brain/spinal cords or from transgenic ALS mice, to generate a more detailed status of the ALS BBB and BSCB. A better appreciation of the status of BBB/BSCB influx transporters in ALS can assist in the design of new chemical entities that can specifically target these influx transporters to enhance CNS exposure of otherwise impermeable drugs. These studies will also highlight, based on their affinity to transporters, which drugs not intended to reach the CNS have increased CNS access in ALS, informing which drugs may require dosage adjustment so as to avoid excessive CNS exposure. Ultimately, this will guide optimum dosing in individuals for all medications consumed by people with ALS to maximise effectiveness (when CNS access is required) and minimise CNS toxicity (when CNS access is not desirable), overall enhancing optimum use of medicines in individuals with ALS.

\section{REFERENCES}

Abbott NJ, Patabendige AA, Dolman DE, Yusof SR, \& Begley DJ (2010). Structure and function of the blood-brain barrier. Neurobiol Dis 37: 13-25.

Abrahao A, Meng Y, Llinas M, Huang Y, Hamani C, Mainprize T, et al. (2019). First-in-human trial of blood-brain barrier opening in amyotrophic lateral sclerosis using MR-guided focused ultrasound. Nat Commun 10: 4373.

Al-Bachari S, Naish JH, Parker GJM, Emsley HCA, \& Parkes LM (2020). Blood-brain barrier leakage is increased in Parkinson's disease. Front Physiol 11: 593026.

Andjus PR, Bataveljić D, Vanhoutte G, Mitrecic D, Pizzolante F, Djogo N, et al. (2009). In vivo morphological changes in animal models of amyotrophic lateral sclerosis and Alzheimer's-like disease: MRI approach. Anat Rec 292: 1882-1892.

Bartels AL, Willemsen AT, Kortekaas R, de Jong BM, de Vries R, de Klerk O, et al. (2008). Decreased blood-brain barrier P-glycoprotein function in the progression of Parkinson's disease, PSP and MSA. J 
Neural Transm 115: 1001-1009.

Beghi E, Mennini T, Bendotti C, Bigini P, Logroscino G, Chiò A, et al. (2007). The heterogeneity of amyotrophic lateral sclerosis: a possible explanation of treatment failure. Curr Med Chem 14:3185-3200.

Bruijn LI, Becher MW, Lee MK, Anderson KL, Jenkins NA, Copeland NG, et al. (1997). ALS-linked SOD1 mutant G85R mediates damage to astrocytes and promotes rapidly progressive disease with SOD1-containing inclusions. Neuron 18: 327-338.

Chan GNY, Evans RA, Banks DB, Mesev EV, Miller DS, \& Cannon RE (2017). Selective induction of Pglycoprotein at the CNS barriers during symptomatic stage of an ALS animal model. Neurosci lett 639:103113.

Chiò A, Logroscino G, Hardiman O, Swingler R, Mitchell D, Beghi E, et al. (2009). Prognostic factors in ALS: A critical review. Amyotroph Lateral Scler 10: 310-323.

Davis TP, Sanchez-Covarubias L, \& Tome ME (2014). P-glycoprotein trafficking as a therapeutic target to optimize CNS drug delivery. Adv Pharmacol 71: 25-44.

Donnenfeld H, Kascsak RJ, \& Bartfeld H (1984). Deposits of IgG and C3 in the spinal cord and motor cortex of ALS patients. J Neuroimmunol 6: 51-57.

Engelhardt JI, \& Appel SH (1990). IgG reactivity in the spinal cord and motor cortex in amyotrophic lateral sclerosis. Arch Neurol 47:1210-1216.

Garbuzova-Davis S, Haller E, Saporta S, Kolomey I, Nicosia SV, \& Sanberg PR (2007). Ultrastructure of blood-brain barrier and blood-spinal cord barrier in SOD1 mice modeling ALS. Brain Res 1157: 126-137.

Garbuzova-Davis S, Hernandez-Ontiveros DG, Rodrigues MCO, Haller E, Frisina-Deyo A, Mirtyl S, et al. (2012). Impaired blood-brain/spinal cord barrier in ALS patients. Brain Res 1469: 114-128.

Garbuzova-Davis S, Saporta S, Haller E, Kolomey I, Bennett SP, Potter H, et al. (2007). Evidence of compromised blood-spinal cord barrier in early and late symptomatic SOD1 mice modeling ALS. PLoS One 2: e1205-e1205.

Ge S, \& Pachter JS (2006). Isolation and culture of microvascular endothelial cells from murine spinal cord. J Neuroimmunol 177:209-214.

Golden PL, \& Pardridge WM (1999). P-glycoprotein on astrocyte foot processes of unfixed isolated human brain capillaries. Brain Res 819: 143-146.

Grande G, Morin L, Vetrano DL, Fastbom J, \& Johnell K (2017). Drug use in older adults with amyotrophic lateral sclerosis near the end of life. Drugs Aging 34: 529-533.

Gurney ME, Pu H, Chiu AY, Dal Canto MC, Polchow CY, Alexander DD, et al. (1994). Motor neuron degeneration in mice that express a human $\mathrm{Cu}, \mathrm{Zn}$ superoxide dismutase mutation. Science 264: 1772-1775.

Han-Xiang D, Hujun J, Ronggen F, Hong Z, Yong S, Erdong L, et al.(2008). Molecular dissection of ALSassociated toxicity of SOD1 in transgenic mice using an exon-fusion approach. Hum Mol Genet 17: 2310-2319.

Henkel JS, Beers DR, Wen S, Bowser R, \& Appel SH (2009). Decreased mRNA expression of tight junction proteins in lumbar spinal cords of patients with ALS. Neurology 72: 1614-1616.

Hobson EV, \& McDermott CJ (2016). Supportive and symptomatic management of amyotrophic lateral sclerosis. Nat Rev Neurol 12: 526-538.

Howland DS, Liu J, She Y, Goad B, Maragakis NJ, Kim B, et al.(2002). Focal loss of the glutamate transporter EAAT2 in a transgenic rat model of SOD1 mutant-mediated amyotrophic lateral sclerosis (ALS). Proc Natl Acad Sci USA 99: 1604-1609. 
Jablonski MR, Jacob DA, Campos C, Miller DS, Maragakis NJ, Pasinelli P, et al. (2012). Selective increase of two $\mathrm{ABC}$ drug efflux transporters at the blood-spinal cord barrier suggests induced pharmacoresistance in ALS. Neurobiol Dis 47: 194-200.

Jablonski MR, Markandaiah SS, Jacob D, Meng NJ, Li K, Gennaro V, et al. (2014). Inhibiting drug efflux transporters improves efficacy of ALS therapeutics. ACTN 1: 996-1005.

Jiang J, Zhu Q, Gendron Tania F, Saberi S, McAlonis-Downes M, Seelman A, et al. (2016). Gain of toxicity from ALS/FTD-linked repeat expansions in C9orf72 is alleviated by antisense oligonucleotides targeting GGGGCC-Containing RNAs. Neuron 90: 535-550.

Leonardi A, Abbruzzese G, Arata L, Cocito L, \& Vische M (1984). Cerebrospinal fluid (CSF) findings in amyotrophic lateral sclerosis. J Neurol 231: 75-78.

Longinetti E, \& Fang F (2019). Epidemiology of amyotrophic lateral sclerosis: an update of recent literature. Curr Opin Neurol 32:771-776.

Lubberink M, van Assema D, Hendrikse NH, Schuit R, Lammertsma A, \& Van Berckel B (2010). Decreased P-glycoprotein function at the blood-brain barrier in patients with Alzheimer's disease as shown by $\left[{ }^{11} \mathrm{C}\right]-$ verapamil and PET. J Nucl Med 51:443-443.

Mehta DC, Short J, \& Nicolazzo J (2013). Altered brain uptake of therapeutics in a triple transgenic mouse model of Alzheime's Disease. Pharm Res 30: 2868-2879.

Mejzini R, Flynn LL, Pitout IL, Fletcher S, Wilton SD, \& Akkari PA (2019). ALS genetics, mechanisms, and therapeutics: where are we now? Front Neurosci 13.

Meyer T, Kettemann D, Maier A, Grehl T, Weyen U, Grosskreutz J, et al. (2020). Symptomatic pharmacotherapy in ALS: data analysis from a platform-based medication management programme. J Neurol Neurosurg Psychiatry 91: 783-785.

Milane A, Fernandez C, Dupuis L, Buyse M, Loeffler JP, Farinotti R, et al. (2010). P-glycoprotein expression and function are increased in an animal model of amyotrophic lateral sclerosis. Neurosci Lett 472: 166-170.

Miyazaki K, Ohta Y, Nagai M, Morimoto N, Kurata T, Takehisa Y, et al. (2011). Disruption of neurovascular unit prior to motor neuron degeneration in amyotrophic lateral sclerosis. J Neurosci Res 89: 718-728.

Nicaise C, Mitrecic D, Demetter P, De Decker R, Authelet M, Boom A, et al. (2009). Impaired blood-brain and blood-spinal cord barriers in mutant SOD1-linked ALS rat. Brain Res 1301:152-162.

Nicolazzo JA, Charman SA, \& Charman WN (2006). Methods to assess drug permeability across the bloodbrain barrier. J Pharm Pharmacol 58: 281-293.

O'Rourke Jacqueline G, Bogdanik L, Muhammad AKMG, Gendron Tania F, Kim Kevin J, Austin A, et al. (2015). C9orf72 BAC transgenic mice display typical pathologic features of ALS/FTD. Neuron 88:892-901.

Ono S, Imai T, Munakata S, Takahashi K, Kanda F, Hashimoto K, et al. (1998). Collagen abnormalities in the spinal cord from patients with amyotrophic lateral sclerosis. J Neurol Sci 160: 140-147.

Pan W, Banks WA, \& Kastin AJ (1997). Permeability of the blood-brain and blood-spinal cord barriers to interferons. J Neuroimmunol 76: 105-111.

Pan Y, \& Nicolazzo JA (2018). Impact of aging, Alzheimer's disease and Parkinson's disease on the bloodbrain barrier transport of therapeutics. Adv Drug Deliv Rev 135: 62-74.

Peters Owen M, Cabrera Gabriela T, Tran H, Gendron Tania F, McKeon Jeanne E, Metterville J, et al. (2015). Human C9orf72 hexanucleotide expansion reproduces RNA foci and dipeptide repeat proteins but not neurodegeneration in BAC transgenic mice. Neuron 88: 902-909. 
Philips T, \& Rothstein JD (2015). Rodent models of amyotrophic lateral sclerosis. Curr Protoc Pharmacol 69: 5.67.61-65.67.21.

Picher-Martel V, Valdmanis PN, Gould PV, Julien J-P, \& Dupré N (2016). From animal models to human disease: a genetic approach for personalized medicine in ALS. Acta Neuropathol Commun 4: 70.

Puris E, Gynther M, Auriola S, \& Huttunen KM (2020). L-Type amino acid transporter 1 as a target for drug delivery. Pharm Res 37:88-88.

Rosen DR, Siddique T, Patterson D, Figlewicz DA, Sapp P, Hentati A, et al. (1993). Mutations in Cu/Zn superoxide dismutase gene are associated with familial amyotrophic lateral sclerosis. Nature 362: 59-62.

Sasaki S (2015). Alterations of the blood-spinal cord barrier in sporadic amyotrophic lateral sclerosis. Neuropathology 35:518-528.

Shefner J, Heiman-Patterson T, Pioro EP, Wiedau-Pazos M, Liu S, Zhang J, et al. (2020). Long-term edaravone efficacy in amyotrophic lateral sclerosis: Post-hoc analyses of Study 19 (MCI186-19). Muscle Nerve 61: 218-221.

Shelkovnikova TA, Peters OM, Deykin AV, Connor-Robson N, Robinson H, Ustyugov AA, et al. (2013). Fused in sarcoma (FUS) protein lacking nuclear localization signal (NLS) and major RNA binding motifs triggers proteinopathy and severe motor phenotype in transgenic mice. J Biol Chem 288: 25266-25274.

Sweeney MD, Sagare AP, \& Zlokovic BV (2018). Blood-brain barrier breakdown in Alzheimer disease and other neurodegenerative disorders. Nat Rev Neurol 14: 133-150.

Uchida Y, Yagi Y, Takao M, Tano M, Umetsu M, Hirano S, et al.(2020). Comparison of absolute protein abundances of transporters and receptors among blood-brain barriers at different cerebral regions and the blood-spinal cord barrier in humans and rats. Mol Pharm 17: 2006-2020.

van Vliet EA, Iyer AM, Mesarosova L, Çolakoglu H, Anink JJ, van Tellingen O, et al. (2019). Expression and cellular distribution of P-glycoprotein and breast cancer resistance protein in amyotrophic lateral sclerosis patients. J Neuropathol Exp Neurol 79:266-276.

Wang J, Xu G, Li H, Gonzales V, Fromholt D, Karch C, et al.(2005). Somatodendritic accumulation of misfolded SOD1-L126Z in motor neurons mediates degeneration: $\alpha \mathrm{B}$-crystallin modulates aggregation. Hum Mol Genet 14: 2335-2347.

Waters S, Swanson MEV, Dieriks BV, Zhang YB, Grimsey NL, Murray HC, et al. (2021). Blood-spinal cord barrier leakage is independent of motor neuron pathology in ALS. Acta Neuropathol Commun 9: 144.

Wegorzewska I, Bell S, Cairns NJ, Miller TM, \& Baloh RH (2009). TDP-43 mutant transgenic mice develop features of ALS and frontotemporal lobar degeneration. Proc Natl Acad Sci USA 106: 18809-18814.

Winkler EA, Sengillo JD, Sullivan JS, Henkel JS, Appel SH, \& Zlokovic BV (2013). Blood-spinal cord barrier breakdown and pericyte reductions in amyotrophic lateral sclerosis. Acta Neuropathol 125:111-120.

Wong PC, Pardo CA, Borchelt DR, Lee MK, Copeland NG, Jenkins NA, et al. (1995). An adverse property of a familial ALS-linked SOD1 mutation causes motor neuron disease characterized by vacuolar degeneration of mitochondria. Neuron 14: 1105-1116.

Yamadera M, Fujimura H, Inoue K, Toyooka K, Mori C, Hirano H, et al. (2015). Microvascular disturbance with decreased pericyte coverage is prominent in the ventral horn of patients with amyotrophic lateral sclerosis. Amyotroph Lateral Scler Frontotemporal Degener 16:393-401.

Zhong Z, Deane R, Ali Z, Parisi M, Shapovalov Y, O'Banion MK, et al. (2008). ALS-causing SOD1 mutants generate vascular changes prior to motor neuron degeneration. Nat Neurosci 11: 420-422.

Zoccolella S, Beghi E, Palagano G, Fraddosio A, Guerra V, Samarelli V, et al. (2007). Riluzole and amyotrophic lateral sclerosis survival: a population-based study in southern Italy. Eur J Neurol 14: 262-268. 